\title{
H. Maurice Goodman: Basic Medical Endocrinology, 4th edn
}

\author{
Academic Press, New York, NY, 2009
}

\author{
M. Susan Smith
}

Received: 12 November 2008/Accepted: 12 November 2008/Published online: 6 February 2009

(C) Humana Press 2009

The 4th edition of this highly successful textbook continues to have the same focus as previous editions--the integrative and regulatory roles of the endocrine systems, with upto-date coverage of important advances in cellular and molecular mechanisms. The integrative focus of this book reflects the over 40 years of experience of the editor with teaching physiology and endocrinology to medical students. It is precisely the integrative focus that makes this textbook so valuable. The first chapters provide an overview of the principles of hormone structure and function and the roles of the primary endocrine glands. The remaining chapters deal with how hormones serve to regulate complex physiological functions, such as fuel metabolism, regulation of salt and water balance, calcium balance, growth and reproduction. These chapters provide an integrative framework for understanding how these functions respond to challenges from the internal or external environment.

Immediately noticeable in the 4th edition is the rich look of the book with its new color figures (300 total) that make many of the illustrations easier to understand. This book continues to be very readable, concise and provides sufficient basic knowledge of how a particular system works without getting bogged down in the unmanageable details of intracellular signaling cascades or regulation of gene transcription. It provides the framework on which a student can add more complex detail of a specific cell or tissue or of a hormone-related disease. An important change in this book is the increase in coverage of the regulation of fuel metabolism, which is covered in three chapters: Hormones of the GI Tract (completely new chapter), The Pancreatic Islets, and Hormonal Regulation of Fuel Metabolism. This increase in coverage is appropriate and reflects the very strong emphasis on this area of research over the past 10 years. The increase in obesity and diabetes in our population has provided the stimulus for this research, which has led to a sizable growth in knowledge of this very complex area. Revisions of other chapters capture most new advances, without greatly expanding the length of the text. However, managing this feat requires leaving out some information. For example, there is no mention of the discovery of kisspeptin, which is made in neurons in the hypothalamus. Kisspeptin is now considered to be the primary regulator of $\mathrm{GnRH}$ neurons and reproductive function. Also, it is likely that a future edition will consider adipose tissue as an endocrine organ, reflecting the array of hormones synthesized by adipocytes and its importance in glucose homeostasis and the metabolic syndrome.

Overall, the text book is essential for medical students. It also can serve as a valuable resource for an upper level undergraduate course or a first year graduate course. 